TITLE:

ON CHAETOGNATHS AND APPENDICULARIANS COLLECTED BY MR. Z. SAGARA IN THE ARAFURA SEA IN MAY-AUGUST 1955

$\operatorname{AUTHOR(S):~}$

Tokioka, Takasi

CITATION:

Tokioka, Takasi. ON CHAETOGNATHS AND APPENDICULARIANS COLLECTED BY MR. Z. SAGARA IN THE ARAFURA SEA IN MAY-AUGUST 1955. PUBLICATIONS OF THE SETO MARINE BIOLOGICAL LABORATORY 1956, 5(2): 203-208

ISSUE DATE:

1956-06-30

URL:

http://hdl.handle.net/2433/174557

RIGHT: 


\title{
ON CHAETOGNATHS AND APPENDICULARIANS COLLECTED \\ BY MR. Z. SAGARA IN THE ARAFURA SEA IN \\ MAY-AUGUST 1955 $^{1)}$
}

\author{
TAKASI TOKIOKA
}

Seto Marine Biological Laboratory, Sirahama

With a Chart and 2 Tables

By courtesy of Mr. Z. SAGARA of the Tôkai Regional Fisheries Research Laboratory, a chance was bestowed fortunately on me to examine fifty-five plankton samples collected by him in the Arafura Sea during his oceanographical observation in the period from the end of May to the begining of August 1955. These samples were hauled by a modified Hensen's net in miniature, $10 \mathrm{~cm}$ in diameter of the mouth, $25 \mathrm{~cm}$ in the maximum diameter, $90 \mathrm{~cm}$ in length and stretched with MülLER's gauze No. 13 (ca. 130 meshes per square inch), from the bottom to the surface at stations shown in Chart 1. The situation of stations, date and time of each sampling and some other data concerning the water of the surveyed area are given at the end of this article, where the haul distance does not mean the depth at respective stations, because the rope may very often be stretched obliquely.

Of these samples, chaetognaths and appendicularians were selected out and identified by myself, while other plankton organisms were submitted to respective specialists for their close examination. In the following, the results of my examination on the two animal groups are given in the form of tables, with some brief notes. Before going further I wish to express my hearty thanks to Mr. Z. SAGARA for his kindness.

\section{Chaetognaths}

Only nine species were found in the following proportion. Their occurrence is shown in detail in Table 1.

Species

1. Sagitta enflata

2. Sagitta bipunctata

3. Sagitta robusta

4. Sagitta bedoti
Number of Individuals

440

1

42

161
Percentage

34

3

13

1) Contributions from the Seto Marine Biological Laboratory, No. 277.

Publ. Seto Mar. Blol. Lab., V (2), 1956. (Article 11) 


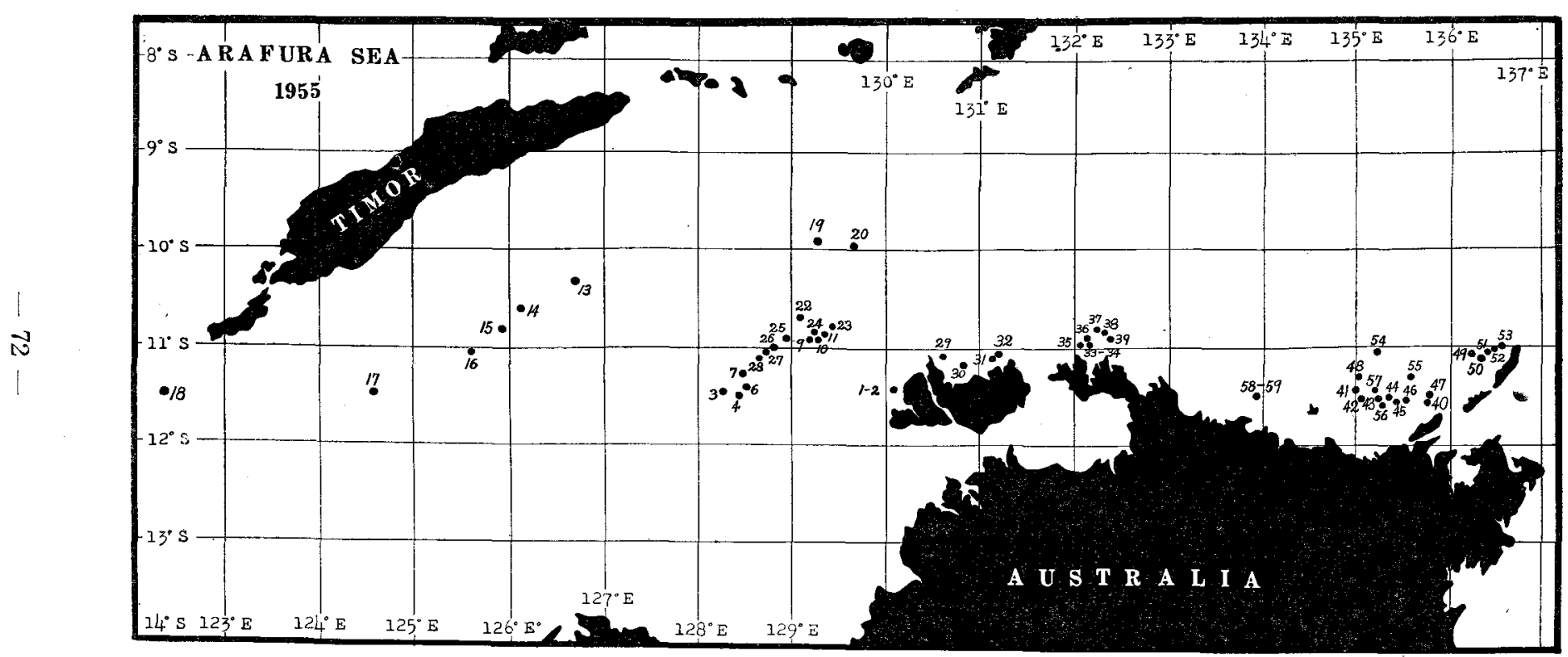

-1
-1
0
0
0
0
0

Chart 1. Map showing stations of sampling. 
5. Sagitta serratodentata pacifica

6

6. Sagitta neglecta 46

4

7. Sagitta regularis

9

1

8. Plerosagitta draco

1

9. Krohnitta pacifica

181

14

10. Damaged individuals or juv.

374

30

1261

Sag. enflata is the commonest species and is followed by Sag. bedoti and Ktta. pacifica. The absence of Sag. hexaptera and the rareness of Sag. serratodentata are considered to be due to the shallowness of the surveyed area where the water was considered, in the period of observation, as being the same as that of the surface layer of the open and deep ocean and quite free from any water masses up-welled from the depth. The universal occurrence of Sag. bedoti is a characteristic in warm coastal waters of the Indian Ocean and the Malay Archipelagoes, and that of Ktta. pacifica and the scarcity of Pterosag. draco were observed also during the survey made by Mr. S. WAJA in 1938. ${ }^{1)}$ Many juvenile individuals, of Ktta. pacifica were found in the present material. The water at Stations 13-18 off the southern coast of Timor was clearly much more oceanic than at other stations and samples hauled at these stations contained only a few number of chaetognaths, including no individual of Sag. bedoti.

\section{Appendicularians}

The following 19 species were detected in the material, the detail of the occurrence of respective species is given in Table 2 .

\section{Species}

1. Oikopleura longicauda

2. Oikopleura fusiformis

3. Oikopleura fusiformis f. cornutogastra

4. Oikopleura intermedia

5. Oikopleura dioica

6. Oikopleura rufescens

7. Oikopleura parva

8. Oikopleura cophocerca

9. Oikopleura spp. (damaged)

10. Megalocercus huxleyi

11. Stegosoma magnum

$\begin{array}{rc}\text { Number of Individuals } & \text { Percentage } \\ 1714 & 52 \\ 107 & 3 \\ 326 & 10 \\ 13 & \\ 68 & 2 \\ 383 & 12 \\ 1 & \\ 30 & 1 \\ 84 & 3 \\ 6 & \\ 5 & \end{array}$

1) TokIOKA, T. (1942): Systematic studies of the plankton organisms occurring in Iwayama Bay, Palao, III. Chaetognaths of the bay and the adjacent waters. Palao Trop. Biol. St. Stud., II (3). 
12. Fritillaria haplostoma 32

13. Fritillaria abjornseni 3

14. Fritillaria arafoera 1

15. Fritillaria formica f. digitata 4

16. Fritillaria pellucida 2

17. Fritillaria borealis f. sargassi (large individual) 5

17a. " " (small individual) 374

18. Fritillaria spp. (damaged) 2

19. Tectillaria fertilis 1

20. Appendicularia sicula 155

21. Kowalevskaia tenuis

Oik. longicauda is the dominant-most species of all and Oik. fusiformis f. cornutogastra, Oik. rufescens and Frit. borealis f. sargassi (small individual) follow it. App. sicula occurs commonly, but not in abundance. Oik. fusiformis, Oik. dioica and Frit. haplostoma occur less frequently and much sparsely. Oik. cophocerca was confined in the present survey to the area west to $129^{\circ} 14^{\prime} \mathrm{E}$. The number of species was more numerous at Sts. 13-18 than at others. Rather universal occurrence of Oik. dioica, though in a small number, seems to show distinctly that the water of the surveyed area is considerably of a neritic nature.

\begin{tabular}{|c|c|c|c|c|c|c|c|c|}
\hline $\begin{array}{l}\text { Station } \\
\text { Number }\end{array}$ & Situation & Date & Time & $\begin{array}{c}\text { Haul } \\
\text { Distance }\end{array}$ & $\begin{array}{l}\text { Surface } \\
\text { Temp. }\end{array}$ & $\begin{array}{l}\text { Bottom } \\
\text { Temp. }\end{array}$ & $\begin{array}{l}\text { Transp- } \\
\text { arency }\end{array}$ & $\begin{array}{l}\text { Water } \\
\text { Colour }\end{array}$ \\
\hline 1 & $\begin{array}{r}11^{\circ} 25^{\prime} \mathrm{S} \\
130^{\circ} 04^{\prime} \mathrm{E}\end{array}$ & $\begin{array}{r}\text { May } \\
29\end{array}$ & $\begin{array}{l}12 \mathrm{~h} \\
10^{\prime}\end{array}$ & $20.5 \mathrm{~m}$ & $29.0 \mathrm{C}$ & $28.4 \mathrm{C}$ & $2.8 \mathrm{~m}$ & 8 \\
\hline 2 & $\begin{array}{r}11^{\circ} 25^{\prime} \mathrm{S} \\
130^{\circ} 04^{\prime} \mathrm{E}\end{array}$ & 30 & $12 \mathrm{~h}$ & 20.0 & 29.2 & 28.4 & 6.0 & 9 \\
\hline 3 & $\begin{array}{r}11^{\circ} 26^{\prime} \mathrm{S} \\
128^{\circ} 16^{\prime} \mathrm{E}\end{array}$ & $\begin{array}{r}\text { June } \\
2\end{array}$ & $\begin{array}{l}14 \mathrm{~h} \\
20^{\prime}\end{array}$ & 40.0 & 29.2 & 28.5 & 10.0 & 7 \\
\hline 4 & $\begin{array}{r}11^{\circ} 28^{\prime} \mathrm{S} \\
128^{\circ} 26^{\prime} \mathrm{E}\end{array}$ & 3 & $\begin{array}{l}8 \mathrm{~h} \\
5^{\prime}\end{array}$ & 33.0 & 28.4 & 28.6 & 11.0 & 7 \\
\hline 6 & $\begin{array}{r}11^{\circ} 25^{\prime} \mathrm{S} \\
128^{\circ} 29^{\prime} \mathrm{E}\end{array}$ & 3 & $\begin{array}{l}13 \mathrm{~h} \\
15^{\prime}\end{array}$ & 36.0 & 28.8 & 28.8 & 14.0 & 7 \\
\hline 7 & $\begin{array}{r}11^{\circ} 18^{\prime} \mathrm{S} \\
128^{\circ} 28^{\prime} \mathrm{E}\end{array}$ & 3 & $\begin{array}{l}15 \mathrm{~h} \\
30^{\prime}\end{array}$ & 37.0 & 28.8 & 28.4 & 15.0 & 7 \\
\hline 9 & $\begin{array}{r}10^{\circ} 58^{\prime} \mathrm{S} \\
129^{\circ} 08^{\prime} \mathrm{E}\end{array}$ & 4 & $\begin{array}{c}8 \mathrm{~h} \\
15^{\prime}\end{array}$ & 35.0 & 28.6 & 28.5 & 12.0 & 8 \\
\hline 10 & $\begin{array}{r}10^{\circ} 58^{\prime} \mathrm{S} \\
129^{\circ} 12^{\prime} \mathrm{E}\end{array}$ & 4 & $12 \mathrm{~h}$ & 35.0 & 28.6 & 28.6 & 10.0 & 8 \\
\hline 11 & $\begin{array}{r}10^{\circ} 56^{\prime} \mathrm{S} \\
129^{\circ} 14^{\prime} \mathrm{E}\end{array}$ & 4 & $\begin{array}{c}16 \mathrm{~h} \\
5^{\prime}\end{array}$ & 33.0 & 28.5 & 28.4 & 14.0 & 8 \\
\hline 13 & $\begin{array}{r}10^{\circ} 21^{\prime} \mathrm{S} \\
126^{\circ} 40^{\prime} \mathrm{E}\end{array}$ & 14 & $\begin{array}{r}7 \mathrm{~h} \\
45^{\prime}\end{array}$ & 33.0 & 28.2 & 28.3 & 33.0 & 3 \\
\hline 14 & $\begin{array}{r}10^{\circ} 39^{\prime} \mathrm{S} \\
126^{\circ} 04^{\prime} \mathrm{E}\end{array}$ & 15 & $\begin{array}{r}9 \mathrm{~h} \\
20^{\prime}\end{array}$ & 26.0 & 27.9 & 27.9 & 20.0 & 4 \\
\hline
\end{tabular}




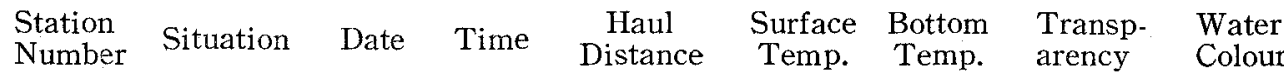

\begin{tabular}{|c|c|c|c|c|c|c|c|}
\hline 15 & $\begin{array}{r}10^{\circ} 47^{\prime} \mathrm{S} \\
125^{\circ} 51^{\prime} \mathrm{E}\end{array}$ & $\begin{array}{r}\text { June } \\
16\end{array}$ & $\begin{array}{l}8 \mathrm{~h} \\
3^{\prime}\end{array}$ & $27.0 \mathrm{~m}$ & $27.9 \mathrm{C}$ & $27.9 \mathrm{C}$ & $20.0 \mathrm{~m}$ \\
\hline 16 & $\begin{array}{r}11^{\circ} 05^{\prime} \mathrm{S} \\
125^{\circ} 34^{\prime} \mathrm{E}\end{array}$ & 16 & $16 \mathrm{~h}$ & 25.0 & 28.2 & 28.2 & 25.0 \\
\hline 17 & $\begin{array}{c}11^{\circ} 26^{\prime} \mathrm{S} \\
124^{\circ} 39.5^{\prime} \mathrm{E}\end{array}$ & 17 & $\begin{array}{r}8 \mathrm{~h} \\
10^{\prime}\end{array}$ & 30.0 & 27.8 & 27.9 & 28.0 \\
\hline 18 & $\begin{array}{c}11^{\circ} 32.5^{\prime} \mathrm{S} \\
123^{\circ} 35^{\prime} \mathrm{E}\end{array}$ & 18 & $\begin{array}{c}9 \mathrm{~h} \\
30^{\prime}\end{array}$ & 30.0 & 28.0 & 28.0 & 30.0 \\
\hline 19 & $\begin{array}{r}9^{\circ} 53^{\prime} \mathrm{S} \\
129^{\circ} 15^{\prime} \mathrm{E}\end{array}$ & 26 & $9 \mathrm{~h}$ & 11.0 & 27.6 & 27.6 & 11.0 \\
\hline 20 & $\begin{array}{r}9^{\circ} 54.5^{\prime} \mathrm{S} \\
129^{\circ} 33.5^{\prime} \mathrm{E}\end{array}$ & 26 & $13 \mathrm{~h}$ & 26.0 & 27.3 & 27.2 & 20.0 \\
\hline 22 & $\begin{array}{c}10^{\circ} 41^{\prime} \mathrm{S} \\
129^{\circ} 03.5^{\prime} \mathrm{E}\end{array}$ & 27 & $\begin{array}{c}7 \mathrm{~h} \\
32^{\prime}\end{array}$ & 40.0 & 27.4 & 27.4 & 11.0 \\
\hline 23 & $\begin{array}{r}10^{\circ} 41.5^{\prime} \mathrm{S} \\
129^{\circ} 16.5^{\prime} \mathrm{E}\end{array}$ & 27 & $17 \mathrm{~h}$ & 38.0 & 27.4 & 27.4 & 10.0 \\
\hline 24 & $\begin{array}{r}10^{\circ} 47^{\prime} \mathrm{S} \\
129^{\circ} 10^{\prime} \mathrm{E}\end{array}$ & 28 & $\begin{array}{r}7 \mathrm{~h} \\
55^{\prime}\end{array}$ & 35.0 & 27.5 & 27.4 & 12.0 \\
\hline 25 & $\begin{array}{r}10^{\circ} 53^{\prime} \mathrm{S} \\
128^{\circ} 51^{\prime} \mathrm{E}\end{array}$ & 28 & $\begin{array}{l}15 \mathrm{~h} \\
50^{\prime}\end{array}$ & 35.0 & 27.6 & 27.6 & 17.0 \\
\hline 26 & $\begin{array}{c}11^{\circ} 00^{\prime} \mathrm{S} \\
128^{\circ} 44.5^{\prime} \mathrm{E}\end{array}$ & 29 & $\begin{array}{c}8 \mathrm{~h} \\
22^{\prime}\end{array}$ & 35.0 & 27.7 & 27.7 & 11.0 \\
\hline 27 & $\begin{array}{c}11^{\circ} 02.5^{\prime} \mathrm{S} \\
128^{\circ} 44^{\prime} \mathrm{E}\end{array}$ & 29 & $\begin{array}{l}16 \mathrm{~h} \\
45^{\prime}\end{array}$ & 36.0 & 27.8 & 27.8 & 15.0 \\
\hline 28 & $\begin{array}{r}11^{\circ} 06^{\prime} \mathrm{S} \\
128^{\circ} 25^{\prime} \mathrm{E}\end{array}$ & $\begin{array}{r}\text { July } \\
1\end{array}$ & $\begin{array}{r}7 \mathrm{~h} \\
15^{\prime}\end{array}$ & 44.0 & 27.2 & 27.2 & 12.0 \\
\hline 29 & $\begin{array}{r}11^{\circ} 04^{\prime} \mathrm{S} \\
130^{\circ} 34^{\prime} \mathrm{E}\end{array}$ & 12 & $\begin{array}{l}14 \mathrm{~h} \\
55^{\prime}\end{array}$ & 38.0 & 26.2 & 26.2 & 5.0 \\
\hline 30 & $\begin{array}{c}11^{\circ} 11.5^{\prime} \mathrm{S} \\
130^{\circ} 45^{\prime} \mathrm{E}\end{array}$ & 13 & $\begin{array}{r}7 \mathrm{~h} \\
55^{\prime}\end{array}$ & 22.0 & 26.1 & 26.1 & 2.0 \\
\hline 31 & $\begin{array}{r}11^{\circ} 07^{\prime} \mathrm{S} \\
131^{\circ} 09^{\prime} \mathrm{E}\end{array}$ & 13 & $15 \mathrm{~h}$ & 23.0 & 26.0 & 26.0 & 1.2 \\
\hline 32 & $\begin{array}{l}11^{\circ} 06.5^{\prime} \mathrm{S} \\
131^{\circ} 10^{\prime} \mathrm{E}\end{array}$ & 14 & $\begin{array}{c}8 \mathrm{~h} \\
10^{\prime}\end{array}$ & 24.0 & 25.0 & 25.0 & 2.0 \\
\hline 33 & $\begin{array}{c}10^{\circ} 59^{\prime} \mathrm{S} \\
132^{\circ} 06.5^{\prime} \mathrm{E}\end{array}$ & 14 & $\begin{array}{l}17 \mathrm{~h} \\
10^{\prime}\end{array}$ & 29.0 & 25.4 & 25.4 & 4.0 \\
\hline 34 & $\begin{array}{c}10^{\circ} 59^{\prime} \mathrm{S} \\
132^{\circ} 06.5^{\prime} \mathrm{E}\end{array}$ & 15 & $\begin{array}{l}8 \mathrm{~h} \\
30^{\prime}\end{array}$ & 29.0 & 25.4 & 25.4 & 6.0 \\
\hline 35 & $\begin{array}{c}10^{\circ} 59.5^{\prime} \mathrm{S} \\
132^{\circ} 01^{\prime} \mathrm{E}\end{array}$ & 15 & $11 \mathrm{~h}$ & 35.0 & 25.6 & 25.5 & 10.0 \\
\hline 36 & $\begin{array}{r}10^{\circ} 55^{\prime} \mathrm{S} \\
132^{\circ} 06^{\prime} \mathrm{E}\end{array}$ & 16 & $\begin{array}{l}10 \mathrm{~h} \\
10^{\prime}\end{array}$ & 37.0 & 25.6 & 25.6 & 5.0 \\
\hline 37 & $\begin{array}{c}10^{\circ} 50.5^{\prime} \mathrm{S} \\
132^{\circ} 10^{\prime} \mathrm{E}\end{array}$ & 16 & $\begin{array}{l}12 \mathrm{~h} \\
10^{\prime}\end{array}$ & 44.0 & 25.7 & 25.7 & 5.0 \\
\hline 38 & $\begin{array}{r}10^{\circ} 54^{\prime} \mathrm{S} \\
132^{\circ} 13^{\prime} \mathrm{E}\end{array}$ & 16 & $\begin{array}{l}13 \mathrm{~h} \\
50^{\prime}\end{array}$ & 35.0 & 25.8 & 25.7 & 5.0 \\
\hline
\end{tabular}




\begin{tabular}{|c|c|c|c|c|c|c|c|c|}
\hline $\begin{array}{l}\text { Station } \\
\text { Number }\end{array}$ & Situation & Date & Time & $\begin{array}{c}\text { Haul } \\
\text { Distance }\end{array}$ & $\begin{array}{c}\text { Surface } \\
\text { Temp. }\end{array}$ & $\begin{array}{l}\text { Bottom } \\
\text { Temp. }\end{array}$ & $\begin{array}{l}\text { Transp- } \\
\text { arency }\end{array}$ & $\begin{array}{l}\text { Water } \\
\text { Colour }\end{array}$ \\
\hline 39 & $\begin{array}{l}10^{\circ} 57.5^{\prime} \mathrm{S} \\
132^{\circ} 16^{\prime} \mathrm{E}\end{array}$ & $\begin{array}{r}\text { July } \\
16\end{array}$ & $\begin{array}{l}15 \mathrm{~h} \\
15^{\prime}\end{array}$ & $23.0 \mathrm{~m}$ & $25.6 \mathrm{C}$ & $25.6 \mathrm{C}$ & $3.5 \mathrm{~m}$ & 9 \\
\hline 40 & $\begin{array}{r}11^{\circ} 38^{\prime} \mathrm{S} \\
135^{\circ} 48^{\prime} \mathrm{E}\end{array}$ & 18 & $12 \mathrm{~h}$ & 35.0 & & & & 8 \\
\hline 41 & $\begin{array}{l}11^{\circ} 28.5^{\prime} \mathrm{S} \\
135^{\circ} 00^{\prime} \mathrm{E}\end{array}$ & 19 & $\begin{array}{l}11 \mathrm{~h} \\
50^{\prime}\end{array}$ & 36.0 & 25.1 & 25.1 & 10.0 & 7 \\
\hline 42 & $\begin{array}{r}11^{\circ} 29^{\prime} \mathrm{S} \\
135^{\circ} 04^{\prime} \mathrm{E}\end{array}$ & 19 & $15 \mathrm{~h}$ & 36.0 & 25.2 & 25.1 & 10.0 & 8 \\
\hline 43 & $\begin{array}{c}11^{\circ} 29^{\prime} \mathrm{S} \\
135^{\circ} 11.5^{\prime} \mathrm{E}\end{array}$ & 26 & $\begin{array}{l}10 \mathrm{~h} \\
35^{\prime}\end{array}$ & 30.0 & 25.3 & 25.3 & 7.0 & 9 \\
\hline 44 & $\begin{array}{r}11^{\circ} 29^{\prime} \mathrm{S} \\
135^{\circ} 21^{\prime} \mathrm{E}\end{array}$ & 26 & $\begin{array}{l}13 \mathrm{~h} \\
45^{\prime}\end{array}$ & 38.0 & 25.6 & 25.6 & 7.0 & 8 \\
\hline 45 & $\begin{array}{c}11^{\circ} 32^{\prime} \mathrm{S} \\
135^{\circ} 22.5^{\prime} \mathrm{E}\end{array}$ & 26 & $\begin{array}{l}15 \mathrm{~h} \\
30^{\prime}\end{array}$ & 38.0 & 25.6 & 25.6 & & 7 \\
\hline 46 & $\begin{array}{r}11^{\circ} 33^{\prime} \mathrm{S} \\
135^{\circ} 33^{\prime} \mathrm{E}\end{array}$ & 27 & $8 \mathrm{~h}$ & 33.0 & 25.3 & 25.3 & 10.0 & 9 \\
\hline 47 & $\begin{array}{r}11^{\circ} 29.4^{\prime} \mathrm{S} \\
135^{\circ} 48.5^{\prime} \mathrm{E}\end{array}$ & 27 & $\frac{11 \mathrm{~h}}{35^{\prime}}$ & 36.0 & 25.5 & 25.5 & 6.0 & 8 \\
\hline 48 & $\begin{array}{c}11^{\circ} 22^{\prime} \mathrm{S} \\
135^{\circ} 01.5 \mathrm{E}\end{array}$ & 27 & $\begin{array}{l}15 \mathrm{~h} \\
10^{\prime}\end{array}$ & 36.0 & 25.5 & 25.5 & 8.0 & 8 \\
\hline 49 & $\begin{array}{r}11^{\circ} 10^{\prime} \mathrm{S} \\
136^{\circ} 19^{\prime} \mathrm{E}\end{array}$ & 28 & $\begin{array}{c}9 \mathrm{~h} \\
50^{\prime}\end{array}$ & 35.0 & 25.6 & 25.6 & 11.0 & 8 \\
\hline 50 & $\begin{array}{r}11^{\circ} 10.3^{\prime} \mathrm{S} \\
136^{\circ} 22.5^{\prime} \mathrm{E}\end{array}$ & 28 & $\begin{array}{l}11 \mathrm{~h} \\
50^{\prime}\end{array}$ & 31.0 & 25.9 & 25.7 & 9.0 & 9 \\
\hline 51 & $\begin{array}{r}11^{\circ} 07^{\prime} \mathrm{S} \\
136^{\circ} 27^{\prime} \mathrm{E}\end{array}$ & 28 & $\begin{array}{l}13 \mathrm{~h} \\
30^{\prime}\end{array}$ & 31.0 & 26.1 & 25.9 & 4.0 & 9 \\
\hline 52 & $\begin{array}{c}11^{\circ} 04^{\prime} \mathrm{S} \\
136^{\circ} 29.5^{\prime} \mathrm{E}\end{array}$ & 29 & $\begin{array}{r}8 \mathrm{~h} \\
35^{\prime}\end{array}$ & 32.0 & 25.9 & 25.8 & 4.0 & 9 \\
\hline 53 & $\begin{array}{c}11^{\circ} 00^{\prime} \mathrm{S} \\
136^{\circ} 29.5^{\prime} \mathrm{E}\end{array}$ & 29 & $\begin{array}{r}9 \mathrm{~h} \\
52^{\prime}\end{array}$ & 39.0 & 25.9 & 25.9 & 6.0 & 9 \\
\hline 54 & $\begin{array}{c}11^{\circ} 06^{\prime} \mathrm{S} \\
135^{\circ} 13.5^{\prime} \mathrm{E}\end{array}$ & 29 & $\begin{array}{l}13 \mathrm{~h} \\
10^{\prime}\end{array}$ & 38.0 & 25.7 & 25.6 & 11.0 & 7 \\
\hline 55 & $\begin{array}{r}11^{\circ} 25.5^{\prime} \mathrm{S} \\
135^{\circ} 37.5^{\prime} \mathrm{E}\end{array}$ & 30 & $\begin{array}{l}14 \mathrm{~h} \\
45^{\prime}\end{array}$ & 35.0 & 25.7 & 25.6 & 7.0 & 9 \\
\hline 56 & $\begin{array}{r}11^{\circ} 31^{\prime} \mathrm{S} \\
135^{\circ} 14^{\prime} \mathrm{E}\end{array}$ & 31 & $\begin{array}{c}8 \mathrm{~h} \\
30^{\prime}\end{array}$ & 28.0 & 25.5 & 25.5 & 7.0 & 9 \\
\hline 57 & $\begin{array}{l}11^{\circ} 28.5^{\prime} \mathrm{S} \\
135^{\circ} 10^{\prime} \mathrm{E}\end{array}$ & 31 & $\begin{array}{l}10 \mathrm{~h} \\
35^{\prime}\end{array}$ & 36.0 & 25.5 & 25.5 & 8.0 & 9 \\
\hline 58 & $\begin{array}{c}11^{\circ} 31^{\prime} \mathrm{S} \\
133^{\circ} 50.5^{\prime} \mathrm{E}\end{array}$ & Aug. & $\begin{array}{l}13 \mathrm{~h} \\
12^{\prime}\end{array}$ & 28.0 & 25.2 & 25.1 & 10.0 & 7 \\
\hline 59 & $\begin{array}{c}11^{\circ} 31^{\prime} \mathrm{S} \\
133^{\circ} 50.5^{\prime} \mathrm{E}\end{array}$ & 3 & $\begin{array}{r}8 \mathrm{~h} \\
30^{\prime}\end{array}$ & 30.0 & 25.1 & 25.1 & 8.0 & 8 \\
\hline
\end{tabular}




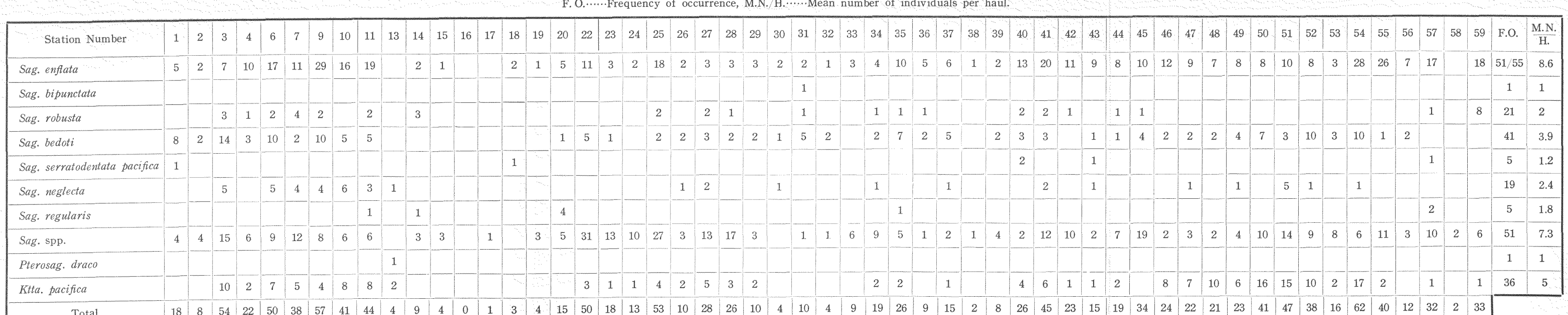
Table 2. Detail of the occurrence of appendicularians.

F. O........Frequency of occurrence, M.N./H.........Mean number of individuals per haul.

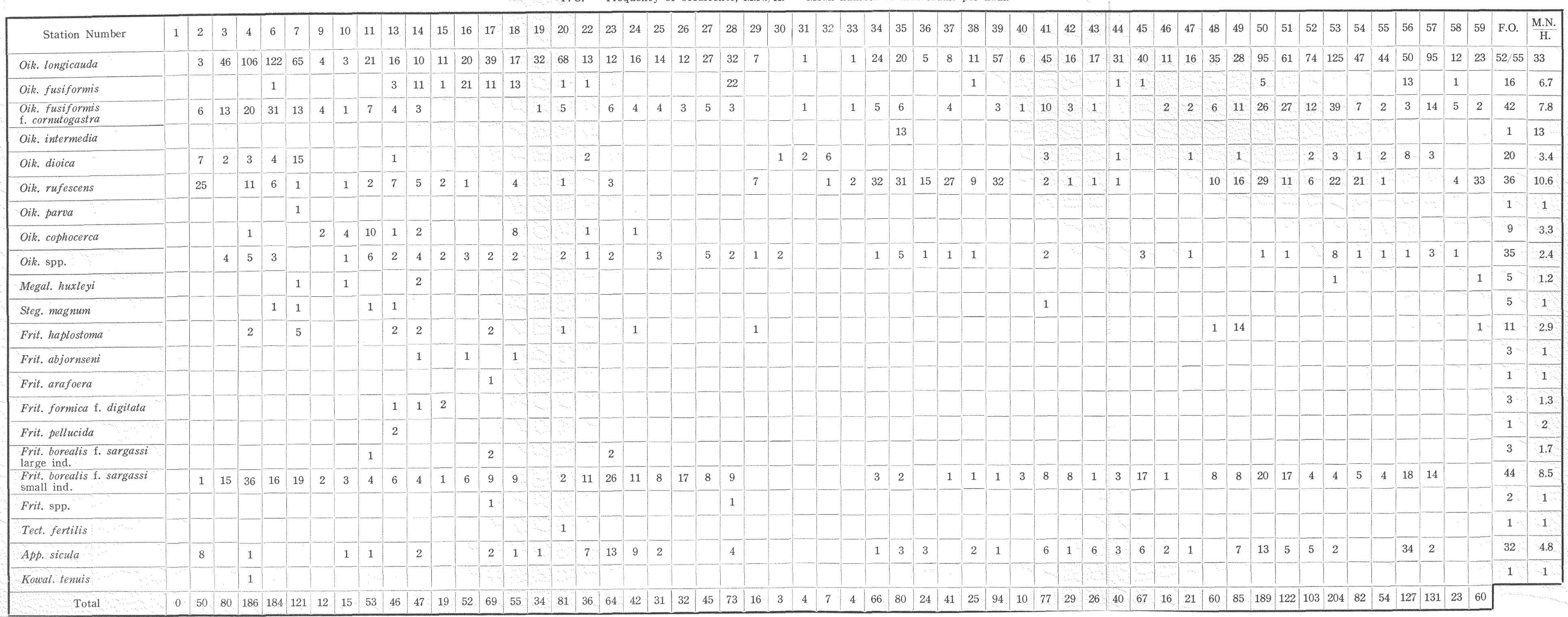

